\title{
Solution Chemistry and Secondary Ion Emission from Amine-Glycerol Solutions
}

\author{
Peter J. Todd \\ Analytical Chemistry Division, Oak Ridge National Laboratory, Oak Ridge, Tennessee, USA
}

\begin{abstract}
Secondary ion mass spectra were obtained from a series of $\mathrm{C}_{4}-\mathrm{C}_{10} n$-alkylamines intro duced via the gas phase onto glycerol. It was found that the amine-characteristic secondary ion intensity varied linearly with amine partial pressure. Henry's law constants and surface activity constants for each of the amines in glycerol solution were measured. A linear correlation was found between amine-characteristic secondary ion intensity and Henry's law concentrations. The concentrations calculated from Henry's law were too low to yield the intensities observed, indicating that secondary ion precursors were not free-base amine molecules but ions in solution. Explicit kinetic equations describing glycerol and amine protonation and deprotonation as a result of primary ion damage to the solutions are derived to rationalize the observed spectra. (f Am Soc Mass Spectrom 1991, 2, 33-44)
\end{abstract}

$\mathrm{F}$ last atom bombardment mass spectrometry (FAB/MS) [1] and liquid secondary ion mass spectrometry (LSIMS) employ secondary ion emission from liquids for ionization of nonvolatile organic solutes. Whatever else can be argued about FAB/MS or LSIMS spectra, there is universal agreement that the chemistry of secondary ion emission from liquids is complex. Nol only is the chemistry of the sputtering event that leads to secondary ion emission difficult to understand, but in and near the sample surface, products formed by damage to the sample from primary particle impact can react with, contaminate, and, in general, alter sample composition [2-5]. The relationship between LSIMS and FAB/MS spectra and the unperturbed sample is thus likely to be a tenuous one. That is, the concentrations of secondary ion precursors and other species in solution near the surface cannot be ascertained with confidence. Connolly and Orth [6], for example, concluded that myriad complications precluded any but fortuitous correlation between spectra and ion concentration in a prepared solution.

There are two major questions about LSIMS that can be answered if the concentration of at least one solute species in the vicinity of the surface can be specified. The first is whether the precursors to positive secondary ions are ions in solution or neutral solute molecules, that is, whether secondary ions are "preformed." The second is whether solute surface activity plays a dominant role in secondary ion emission. These questions have yet to be answered simultaneously for any solute-glycerol solution. This is

Address reprint requests to Peter J. Todd, Analytical Chemistry Division, Oak Ridge National Laboratory, P. O. Box 2008, Oak Ridge, TN 37831-6365. because only the total analytical concentration of neutral and ionic solute can be known and because the ratio of surface to bulk concentrations can be large. Effects of surface adsorption may overwhelm effects of solute protonation, and vice versa. To solve this dilemma, solute concentration must be defined in a way that (1) is independent of primary-ion-induced chemistry, (2) includes a definition of the ratio of adsorbed to dissolved solute concentrations, and (3) permits distinction between ionic and neutral solute.

Solute-solvent systems can be defined any number of ways, for example, by molar or molal concentration of solute, by surface concentration, or by the vapor pressure of a volatile solute above the solution. The last approach is generally not applicable to LSIMS because LSIMS is used mainly for obtaining mass spectra of nonvolatile analytes, although there are exceptions [7]. In terms of sample system definition, however, there are certain advantages to introducing solutes via the gas phase at known pressure onto glycerol. The most important advantage is that the equilibrium concentration of dissolved solute can be defined by Henry's law [8], eq 1 .

$$
P=k_{\mathrm{H}} X
$$

where $P$ is the partial pressure of a particular solute, $k_{\mathrm{H}}$ is the Henry's law constant of the solute in glycerol, and $X$ is the equilibrium mole fraction of solute in solution.

Henry's law is obeyed regardless of the acidity/ basicity or ionic concentration of the solution [8] but can be used only to specify the concentration of neutral solute and necessarily excludes protonated or otherwise ionized solute. The Henry's law conslant is temperature-dependent, but surface temperatures and thermal gradients [9] are believed to be relatively 
unaffected by primary ion current densities less than $1 \mu \mathrm{A} / \mathrm{cm}^{2}$. Thus, within limits, concentration of solute in a glycerol solution can be specified if the partial pressure of the solute and Henry's law constant are known.

The rate at which Henry's law concentration is established in the first $100 \AA$ of glycerol is demonstrably rapid. The mathematics of such diffusion is well known in general; the problem is known as diffusion in a semiinfinite medium [10]. By employing a lower limit for the diffusion coefficient [11] of $10^{-9} \mathrm{~cm}^{2} / \mathrm{s}$, it can be demonstrated that within $20 \mathrm{~ms}$ of establishing a partial pressure, solute concentration within $100 \AA$ of the surface approaches $90 \%$ of its equilibrium value. For a larger diffusion constant, equilibrium is achieved more rapidly.

Adsorption of solute on the solution surface is similarly rapid when the solute is introduced via the gas phase, because adsorption is not limited by diffusion from the bulk. From what is known about adsorption from the gas phase and the kinetic theory of gases, adsorption equilibrium between vapor and surface should occur within $1 \mathrm{~ms}$ for the systems studied here $[12,13]$; solute concentration on and near the surface is thus independent of sample sputtering at low (ca. $1 \mu \mathrm{A} / \mathrm{cm}^{2}$ ) primary ion current density, where the rate of surface removal is only about 4.4 monolayers per second [9].

A number of reports have attributed to solute surface activity a pronounced role in LSIMS spectra obtained from nonvolatile solute-glycerol solutions [14-16]. However, this conclusion is inconsistent with what is known about the rate at which adsorption from solution occurs. For example, in early studies of surface phenomena in water, McBain and Swain determined that hours were required for surface-bulk equilibrium to become established [17]. Diffusion coefficients of even small molecules in glycerol are about four orders of magnitude less than typical diffusion constants in water [18]. It thus seems unlikely that solute surface activity could have a pronounced effect on LSIMS spectra when surface excess concentration must be established via diffusion from the bulk. Nonetheless, to test the importance of solute surface activity on LSIMS spectra, a series of volatile compounds can be chosen so that the variation in measured Henry's law constants among members of the series is different from the variation in surface activity among the members. If secondary ion emission is a function of surface concentration, then measured secondary ion intensity should explicitly vary with surface concentration. By introducing solute via the gas phase, the effect of solute adsorption on secondary ion emission can be ascertained without the complication of solute diffusion from the bulk of solution.

Careful sample system definition can provide insight into another mechanistic ambiguity of LSIMS. Some reported results indicate a pronounced time dependence in LSIMS spectra [2-5, 19], whereas others have implied negligible time dependence [9]. The disparity may be a result of solvent or matrix depletion via preferential evaporation and sputtering, that is, a physical effect [9]. For solute introduced via the gas phase, the inlet system of the mass spectrometer serves as a virtually infinite source of solute for the sample surface and subsurface. As indicated above, the defined concentration of solute is independent of evaporation, sputtering, and time. Thus physical contributions to time dependence should be negligible. Time dependence may also be a result of radiation damage to certain solute species [3-5], that is, a chemical effect. By using solutes from a single compound class, effects of physical properties of each member of the class on secondary ion emission are separated from the myriad unknown chemical effects induced by primary particle bombardment. Presumably, these effects are the same for all members of a compound class. For example, the chemistry of $n$-alkylamines is dominated by the chemistry of the $\mathrm{NH}_{2}$ group; proton affinities of those larger than methylamine are greater than that of glycerol [20].

Low molecular weight $\left(\mathrm{C}_{4}-\mathrm{C}_{10}\right) n$-alkylamines were chosen for this study because they are volatile [21] and can be introduced into the mass spectrometer as gases via an inlet system. It has been demonstrated that emission of secondary ions characteristic of adsorbed or dissolved vapor can be detected when the solute is introduced via the gas phase [7]. When introduced onto a sample of pure glycerol, the concentration of alkylamine in the vicinity of the glycerol surface is determined by Henry's law, eq 1 .

A practical advantage results from introducing solutes via the gas phase. For SIMS in general, reproducible measurement of absolute secondary ion currents from sample to sample is difficult. Even small changes in the shape of samples can have a pronounced effect on measured secondary inn current. Replicate samples from a single solution are likely to show as significant a variation in total secondary ion current as might be observed from samples of different analyte concentration. When the solute is introduced via the gas phase, solute concentration can be altered by simply changing the partial pressure of solute without changing the sample. Furthermore, an initially pure glycerol sample provides a benchmark spectrum and permits measurement of total secondary ion yield. To further improve reproducibility, $\mathrm{Ar}^{+}$ions of measured kinetic energy, current, and current density are employed as primary particles. These important beam parameters can be measured and reproduced from day to day more accurately than can kinetic energy, flux, and flux density of fast atoms used in $\mathrm{FAB} / \mathrm{MS}$.

\section{Experimental}

\section{Mass Spectrometer}

Mass spectral data were obtained by using a Nier-Johnson secondary ion mass spectrometer of local design and construction, described elsewhere 
[22]. The primary ion beam is generated in a discharge source, configured so that all acceleration, extraction, focusing, and deflection potentials are referenced to the secondary ion accelerating potential. The primary ion beam strikes the sample $70^{\circ}$ from target normal. The secondary ion source was operated at 8 $\mathrm{kV}$ accelerating potential, with an extraction field nominally $1000 \mathrm{~V} / \mathrm{cm}$. Detection is by continuous dynode electron multiplier. A dedicated DEC PDP $11 / 23$ is interfaced to the secondary ion source and mass analyzer for control and data acquisition.

\section{Measurement of Primary Ion Current Density}

Primary beam alignment and beam current measurement were accomplished only after stabilization by at least $1 \mathrm{~h}$ of continuous discharge in the primary ion source. Details of the method of alignment and current density measurement are given elsewhere [23]. Basically, a target loaded with $\mathrm{NaI}$ is inserted into the source of the mass spectrometer in place of a normal target. From the light emitted from the target when it is struck by primary ions, primary beam alignment for various primary ion focusing conditions can be determined. This technique is used to ensure that the glycerol sample will be uniformly irradiated. By using a shielded Faraday cup with a known aperture in place of a normal target, primary current density can be measured. Primary focusing conditions can then be adjusted to yield a particular primary ion current density. Unless otherwise indicated, a primary ion current density of $0.5 \mu \mathrm{A} / \mathrm{cm}^{2}$ of 5-keV Ar ${ }^{+}$ions was employed.

\section{Secondary Ion Current Measurements}

Secondary ion current was measured in terms of mass spectrometer detector-preamplifier-amplifier output voltage and was recorded either manually or by the 12-bit analog-to-digital converter of the computer interface. When recorded during repetitive cyclical scanning of the magnetic field of the mass analyzer, secondary ion currents reflect the current integrated over the time a particular ion was being transmitted to the detector. The data collection rate was $5 \mathrm{kHz}$.

Drift in measured secondary ion current from all samples appeared to be monotonic and due solely to changes in the ion optics caused by sample depletion. To mitigate such ion optical effects, source, $\alpha, \beta$, and detector slits were opened fully for the measurement of secondary ion intensities, resulting in a loss in mass resolving power. A neat glycerol LSIMS spectrum shows peaks at every nominal mass. Peaks in the LSIMS spectrum due to amine-characteristic ions are indistinguishable from glycerol-characteristic ions when both have the same nominal mass-to-charge ratio. This is a negligible source of error, because amine-characteristic secondary ions have an even nominal mass, and ions having an even nominal mass are of low abundance in the spectrum of glycerol.
Table 1. Physical properties of amines in glycerol solution

\begin{tabular}{lccc}
\hline Amine & $\begin{array}{c}\mathrm{d} \sigma / \mathrm{dc} \\
\mathrm{L} /(\mathbf{c m} \cdot \mathrm{mol})]\end{array}$ & $\begin{array}{c}k_{\mathrm{H}} \\
\text { (torr })\end{array}$ & $\begin{array}{c}d\left(\mathrm{M}+\mathrm{H}^{+}\right\} / \mathrm{dP} \\
\left(10^{5} \text { torr }^{-1}\right)\end{array}$ \\
\hline \hline Propylamine & -67.1 & 66.0 & \\
Butylamine & -80.2 & 64.1 & \\
Amylamine & -227.3 & 29.2 & 1.4 \\
Hexylamine & -1181.8 & 12.5 & 2.2 \\
Heptylamine & & 6.0 & 4.8 \\
Octylamine & -3830.6 & 2.3 & 7.0 \\
Decylamine & & 0.65 & 30.4 \\
\hline
\end{tabular}

\section{Surface Tension Measurements}

Stock glycerol solutions $(0.5 \mathrm{M})$ of each amine were prepared separately in drybags flooded with argon and were allowed to equilibrate overnight. Weighed aliquots of these solutions were then neutralized with an appropriate volume of $0.1000 \mathrm{~N} \mathrm{HCl}$ and backtitrated with $0.1000 \mathrm{~N} \mathrm{NaOH}$ (bromocresol green indicator) to determine amine concentration. The remainder of each stock solution was used to prepare various solutions of the amines down to $0.001 \mathrm{M}$ as appropriate for the surface tension measurements for each solution. Solutions were prepared by weight because it was impossible to fill a volumetric flask exactly to the fiducial with glycerol owing to its viscosity. The density of each solution was measured with a hydrometer.

Surface tension measurements were made by using a duNuoy balance enclosed in a drybag flooded with dry argon. Temperature was maintained at $25 \pm 2{ }^{\circ} \mathrm{C}$ within the bag. A different drybag was used for each amine, because slight contamination can seriously alter surface tension measurements and the amines are volatile. Plots of surface tension versus amine concentration were obtained. The observed values for the slopes of these plots are listed in Table 1.

\section{Vapor Pressure Measurements}

Attempts to measure amine partial pressure in glycerol solutions by using the method of Christie and Crisp [24] failed to yield reasonable or reproducible results. Consequently, glycerol solutions of each amine were prepared from dry glycerol such that the partial pressure of the amine would be approximately 0.1-2 torr if the solutions were ideal, that is, using Raoult's law and the known vapor pressure of each amine [21]. About $100 \mathrm{~mL}$ of each solution was placed in a separate $250-\mathrm{mL}$ Erlenmeyer flask. Each flask was attached via a ground glass valve to a vacuum line maintained at $2 \times 10^{-2}$ torr. After two freezepump-thaw cycles, the flask was sealed from the vacuum line and the temperature of the solution was raised to $25^{\circ} \mathrm{C}$ by immersing the flask in a water bath. While the flask was being stirred with a magnetic stirring bar, the solution was vented to the vacuum system and the pressure was allowed to reach a constant value as measured by a capacitance 
manometer. This pressure was recorded, and the sample was then removed from the vacuum line. Three weighed aliquots of each solution were neutralized with $0.1000 \mathrm{~N} \mathrm{HCl}$ and back-titrated with 0.1000 $\mathrm{N} \mathrm{NaOH}$ (bromocresol green indicator) to determine amine concentration. Activity coefficients of the various amines in glycerol were less than those measured by Christie and Crisp [24] for the same amines in water, for which solutions substantial positive deviation was reported. Simply put, amine-glycerol solutions are more nearly ideal than amine-water solutions, as expected. Henry's law constants derived from these measurements for amine-glycerol solutions are shown in Table 1.

\section{Inlet System Calibration}

A Bayard-Alpert gauge was attached to a flange constructed so that with the secondary ion source removed, the gauge could be attached to the suurce housing in place of the secondary ion source assembly. With such an arrangement, the $1.9-\mathrm{cm}$ opening of the inverted Bayard-Alpert gauge occupied the position of a normal sample. With the inlet system at 200 ${ }^{\circ} \mathrm{C}$, various quantities of heptane, methanol, and 0 nitrotoluene were injected and the indicated pressure was recorded. For these compounds, the sensitivity of the ion gauge is known, so it was possible to determine the relationship between moles injected into the inlet system and pressure of the species over the secondary ion source target region. A plot of pressure versus moles injected was found to be linear up to a pressure of $10^{-5}$ torr, with a slope of $1.75 \times 10^{-3}$ torr $/ \mathrm{mol}$, for all three reference compounds.

\section{Samples}

Amines were obtained commercially (Aldrich Chemical Co., Milwaukee, WI) with a specified purity as $>97 \%$. All amines were redistilled in glass. Mass spectra of these compounds did not indicate the presence of significant contamination. Glycerol (Aldrich, Gold Label) purity was indicated as $>99.5 \%$, and all samples of glycerol were taken from a single 2-L bottle. Extensive drying and degassing of glycerol was accomplished by heating and stirring it under vacuum for a period of $4 \mathrm{~h}$. This glycerol was maintained under vacuum until used. Failure to completely degas glycerol resulted in extensive boiling of samples when they were inserted into the ion source of the mass spectrometer.

Dry glycerol was loaded onto $0.95-\mathrm{cm}$-diameter stainless steel probe tips via glass transfer pipet and immediately inserted into the vacuum lock of the secondary ion source. In earlier work [9] we reported that glycerol was rapidly depleted during LSIMS experiments. However, by employing the extensive degassing described above and using lower primary ion current densities than before, we observed that sec-

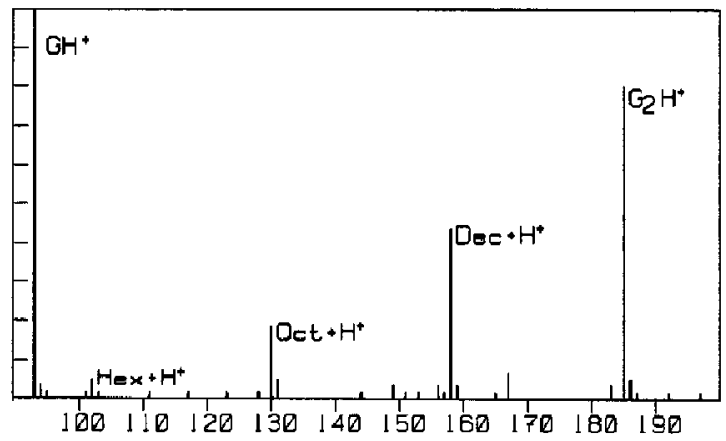

Figure 1. LSIMS spectrum obtained from introduction of a 1:1:1 molar mixture of hexylamine, octylamine, and decylamine onto glycerol under $5-\mathrm{keV} \mathrm{Ar}{ }^{+}\left(0.5 \mu \mathrm{A} / \mathrm{cm}^{2}\right)$ bombardment. Partial pressure of each amine was $1.5 \times 10^{-7}$ torr.

ondary ion emission from glycerol samples was stable for up to $1 \mathrm{~h}$.

\section{Results and Discussion}

Within an experimental error of $\pm 10 \%$, introduction of amines at pressures up to $10^{-6}$ torr had no effect on either total secondary ion current or the rate of drift of total secondary ion current, typically $\pm 1 \% / \mathrm{min}$. Relative abundances of particular ions, enclosed by braces \{\} , are to be taken as the fraction of total secondary ion current carried by the indicated species.

Figure 1 shows a partial LSIMS spectrum of a glycerol solution of hexylamine, octylamine, and decylamine. The solution was prepared by introducing the amines at individual partial pressures of $1.5 \times$ $10^{-7}$ torr during irradiation by $0.5-\mu \mathrm{A} / \mathrm{cm}^{2}, 5.0-\mathrm{keV}$ $\mathrm{Ar}^{+}$. Major peaks arising from the respective amines were protonated amines, $[\mathrm{M}+\mathrm{H}]^{+}$, at $m / z 102$ for hexylamine, $m / z 130$ for octylamine, and $m / z 158$ for decylamine; no amine-specific fragmentation ions of appreciable abundance were observed. With the exception of the amine-characteristic ions, relative abundances of the ions detected were the same as those of a pure glycerol sample.

A substantial difference in the relative intensity of the three protonated amine peaks is apparent in Figure 1, though the partial pressures of the amines were identical. If the amines were protonated in the gas phase, the intensity of the protonated amine peaks would be nearly identical. This is because the rate of exothermic gas-phase protonation is a linear function of the collision frequency, which varies linearly with pressure and is only a weak function of amine mass [25]. Thus, the detected secondary ions apparently originate in the glycerol sample.

To further test this assertion, kinetic energy distributions were obtained by sweeping the secondary ion source accelerating/focusing voltages. Such distributions for the protonated amines were found to be similar to those of secondary proton-bound glycerol 
dimers, trimers, etc., and narrower than that of protonated glycerol. The low-energy "tail" of the protonated glycerol ion distribution reflects its formation by metastable decomposition of higher mass ions in the $1000 \mathrm{~V} / \mathrm{cm}$ extraction field of the secondary ion source. If protonated amine were formed in the region between the sample surface and the secondary ion extraction element, a similar tail would be expected.

\section{Secondary Ion Intensity and Amine Pressure}

The relative intensities of the protonated amines shown in Figure 1 correlate with alkyl chain length in a qualitative way, as do many physical properties. It can be seen from Table 1, for example, that there is a decrease in Henry's law constants and an increase in surface activity with increasing alkyl chain length. It is not clear from Figure 1 what gives rise to the relative abundances of the protonated amine peaks. Definition of a four-component, three-phase (vapor, surface, and bulk) mixture is complicated. To simplify definition of the system and analysis of data, the remainder of this work is devoted to the behavior of individual two-component systems. As mentioned earlier, for each amine-glycerol system, the spectrum and total ion current from the initially pure glycerol sample can be obtained as a reference before any solute is introduced. Changes in LSIMS spectra effected by gaseous introduction of each amine can be normalized to this reference and used to compare the effects of each amine.

The most likely physical properties to affect secondary ion emission are those that, for a given solute pressure, affect surface and bulk solute concentration. These physical properties are solute surface activity and Henry's law constant. As can be seen from Table 1 , the functional relationships between these properties and chain length are different. To ascertain if solute surface activity dominates secondary ion emission, it is necessary to show an explicit correlation between excess surface concentration and secondary ion intensity that exceeds experimental error. Whereas it is impractical to measure surface tension and solute surface concentration within the mass spectrometer ion source, excess concentration can be determined from eq 2, attributed to Gibbs:

$$
\Gamma=-\frac{C}{R T} \frac{d \sigma}{d C}=-\frac{X}{R T} \frac{d \sigma}{d X}
$$

where $\Gamma$ is the equilibrium surface excess concentration $\left(\mathrm{mol} / \mathrm{m}^{2}\right), C$ and $X$ are solute concentration and mole fraction, respectively; $R$ is the gas constant, $T$ is the temperature of the solution; and $d \sigma / d C$ and $d \sigma / d X$ are the changes in solution surface tension $(\mathrm{N} / \mathrm{m})$ with surfactant concentration and mole fraction, respectively.

Equation 3, generated by substitution of eq 1 into

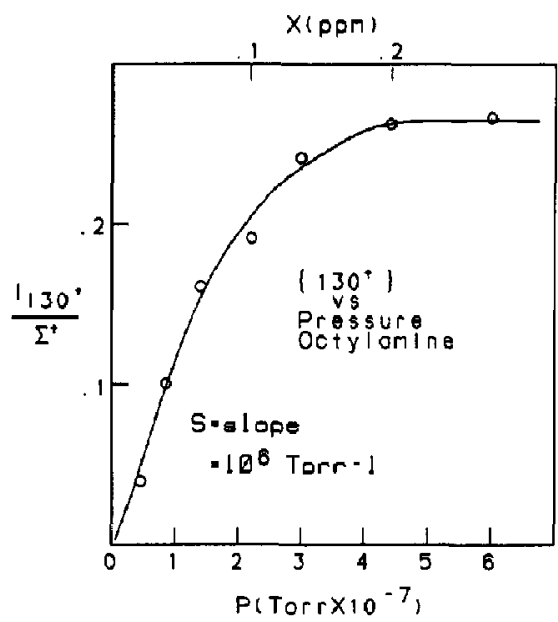

Figure 2. Relative intensity of secondary protonated octylamine as a function of partial pressure (lower abscissa) and Henry's law octylamine concentration (upper abscissa). The slope $S$ refers to the linear portion of the plot.

eq 2, is more convenient to work with here.

$$
\Gamma=-\left(\frac{P}{k_{\mathrm{H}}}\right)\left(\frac{1}{R T}\right) \frac{d \sigma}{d \mathrm{X}}
$$

From eqs 3 and 1 , it is clear that both $\Gamma$ and $X$ are related to partial amine pressure by constants. The appropriate constants are listed in Table 1.

Evaluation of the constants in Table 1 is instructive. The surface of a glycerol droplet contains about $4 \times$ $10^{14}$ molecules $/ \mathrm{cm}^{2}$ or $6.6 \times 10^{-10} \mathrm{~mol} / \mathrm{cm}^{2}$. Since $\Gamma$ is also in terms of $\mathrm{mol} / \mathrm{cm}^{2}$, this surface concentration can be used to calculate the surface mole fraction of an adsorbed species in equilibrium with a bulk solution. For example, at equilibrium, the surface mole fraction of octylamine is almost 3200 times the bulk mole fraction. For amylamine, the ratio of mole fractions is only about 200 . If the relative protonated amine intensities from solutions of octylamine and amylamine having the same Henry's law concentrations are equal, secondary ion emission is governed by bulk amine concentration. If, on the other hand, the intensity of protonated octylamine is 16 $(=3200 / 200)$ times that of protonated amylamine, then secondary ion emission better reflects surface excess concentration.

Figure 2 is a plot of $\left\{130^{+}\right\}$as a function of octylamine partial pressure. For a pressure range up to $2 \times 10^{-7}$ torr and relative abundance up to about 0.2 , $\left\{130^{+}\right\}$is a linear function of pressure. At higher pressures, $\left\{130^{+}\right\}$is independent of amine pressure. For all the amines studied, plots of protonated amine intensity versus pressure showed the same general shape as that of Figure 2. Similar plots of intensity versus concentration have been reported for nonvolatile analytes in glycerol [6] and sulfuric acid [26].

Equations 1 and 3 predict linear relationships be- 


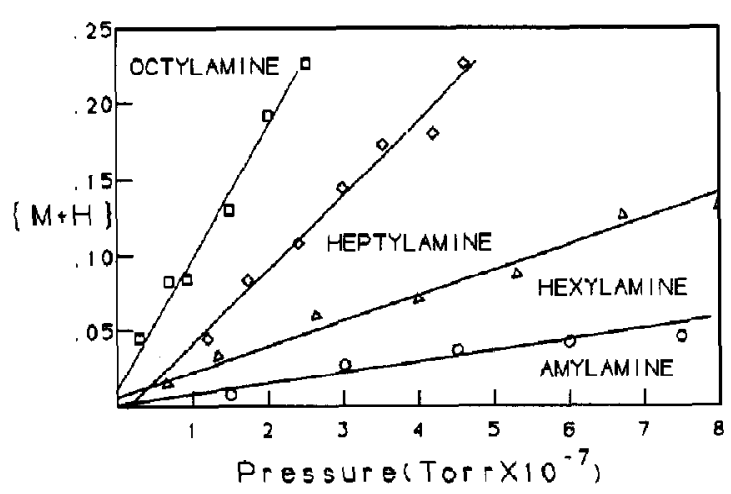

Figure 3. Plots of secondary protonated amine relative intensity versus pressure for various amines studied.

tween bulk and surface concentrations, respectively, and partial pressure, but only at low pressure. Nonlinear behavior at higher pressure (and concentration) is expected for both eqs 1 and 3 , because of solution nonidealities and surface saturation, respectively. Exactly why nonlinear behavior is observed at higher pressure for plots such as Figure 2 is not clear. However, to avoid inclusion of nonlinear effects, further discussion will be limited to amine partial pressures such that surface and bulk concentrations and secondary ion intensity are linear with pressure. Linear portions of plots of secondary ion relative intensity versus partial pressure of various amines are shown in Figure 3.

Figure 4 shows the dependence of measured secondary protonated amine relative intensity on (a) Henry's law mole fraction and (b) surface concentration for selected amines. In Figure 4a, all data points, regardless of molecular weight, fall along the same plot; those of Figure $4 \mathrm{~b}$ do not. However, both plots of Figure 4 show a correlation of sorts, because the limit of useful partial pressures is different for each amine, forcing most of the data for the plots to be close to the origin.

A more definitive test for quantitative correlation is by differential analysis. It is evident from Figure 3 that slopes of protonated amine relative intensity versus pressure are distinctly different; for the various amines; furthermore, the plots are linear. Defining $S$ as the slope of any particular plot in Figure 3 leads to eqs $4 a$ and $4 b$, based on the assumption that $S$ is a function of either bulk concentration or surface concentration, respectively.

$$
S=\frac{d\left\{\mathrm{M}+\mathrm{H}^{+}\right\}}{d P}=\frac{d\{\mathrm{M}+\mathrm{H}\}}{d X} \frac{d X}{d P}
$$

or

$$
S=\frac{d\left\{\mathrm{M}+\mathrm{H}^{+}\right\}}{d P}=\frac{d\left\{\mathrm{M}+\mathrm{H}^{+}\right\}}{d \Gamma} \frac{d \Gamma}{d P}
$$

Because the chemistry of the amines is presumably

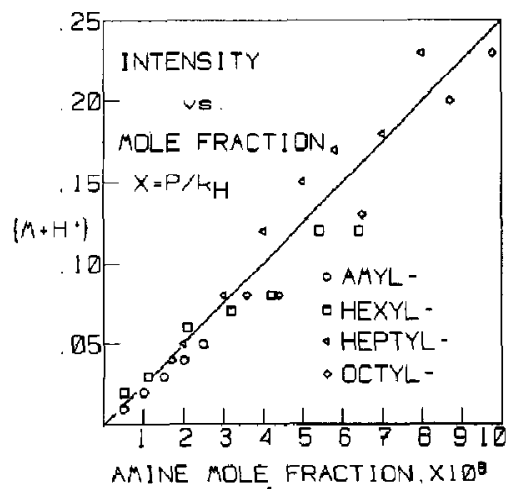

A

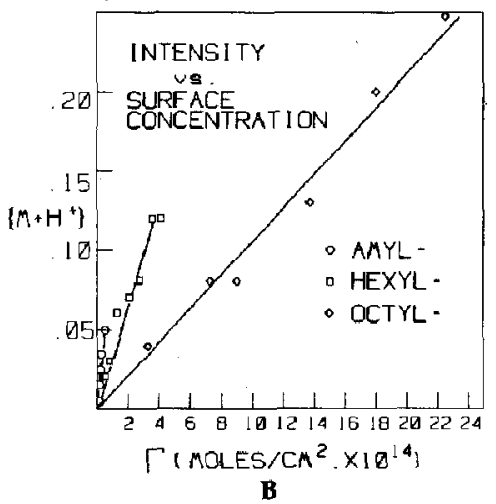

Figure 4. Relative intensity of various protonated amines versus (a) calculated Henry's law concentrations and (b) calculated surface concentrations.

identical, it seems reasonable that whether eq $4 \mathrm{a}$ or $4 \mathrm{~b}$ is the correct expression, $d\left\{\mathrm{M}+\mathrm{H}^{+}\right\} / d X$ or $d\{\mathrm{M}+$ $\left.\mathrm{H}^{+}\right\} / d \Gamma$, respectively, is independent of amine identity. This implies that the latter terms in eqs $4 a$ and $4 b$ cause differences in sensitivity. Equations $1 \mathrm{a}$ and $3 a$ are the differential equivalents of eqs 1 and 3 that describe bulk and surface concentrations, respectively.

$$
\begin{gathered}
\frac{d X}{d P}=\frac{1}{k_{\mathrm{H}}} \\
\frac{d \Gamma}{d P}=\left(\frac{1}{k_{\mathrm{H}}}\right)\left(\frac{1}{R T}\right) \frac{-d \sigma}{d X}
\end{gathered}
$$

For each amine, $d X / d P$ and $d \Gamma / d P$ are both unique and constant, and their values can be obtained from the data in Table 1 . Since either $d X / d P$ or $d \Gamma / d P$ is the presumed cause of difference in sensitivity among the amines, either a plot of $S$ versus $k_{\mathrm{H}}^{-1}$ will yield a straight line or a plot of $S$ versus $k_{\mathrm{H}}^{-1}(-d \sigma / d X)$ will. If the former is true, then the concentration of amine in the bulk determines secondary ion intensity. If the latter is true, then the concentration of solute on the surface determines secondary ion intensity. Both cannot be true. Figure 5a shows a plot of sensitivity versus $1 / k_{H}$ that indicates a linear relationship be- 


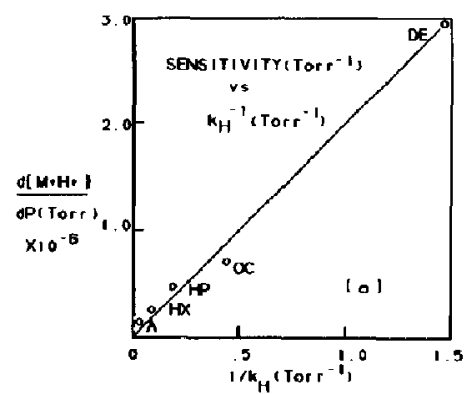

A

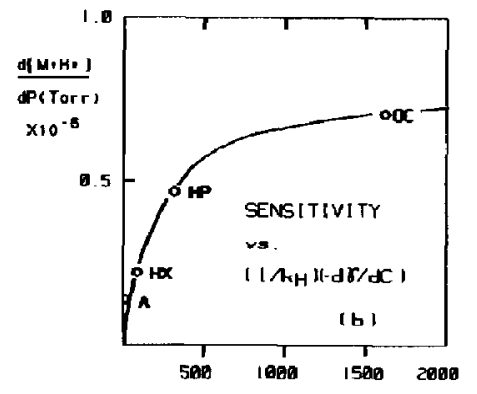

I I / $\left.\mathrm{kH}_{\mathrm{H}}\right)$ l-do/dC II düne/cm-n-Torr I

B

Figure 5. Sensitivity of LSIMS $\left(d\left\{\mathrm{M}+\mathrm{H}^{+}\right\} / d P\right)$ plotted (a) as a function of inverse Henry's law constants and (b) as a function of $1 / k_{\mathrm{H}}(-d \sigma / d C) . \mathrm{A}=$ amylamine, $\mathrm{HX}=$ hexylamine, $\mathrm{HP}$ $=$ heptylamine, $O C=$ octylamine, $D E=$ decylamine (surface activity data not available for $\mathrm{DE}$ ).

tween sensitivity and $1 / k_{H}$. Figure $5 \mathrm{~b}$ is a plot of sensitivity versus $1 / k_{\mathrm{H}}(-d \sigma / d C)$. It is clearly evident that the plot in Figure 5a is linear whereas the plot in Figure $5 b$ is not. Explicitly, this means that eq $4 b$ is invalid, and sensitivity is determined by eq 4 .

$$
\frac{d\left\{\mathrm{M}+\mathrm{H}^{+}\right\}}{d P}=\frac{1}{k_{\mathrm{H}}} \frac{d\left\{\mathrm{M}+\mathrm{H}^{+}\right\}}{d \mathrm{X}}=S
$$

Henry's law constants from amylamine to decylamine vary by a factor of almost 50 . For the partial pressures of amines studied, bulk amine concentration was varied by over two orders of magnitude and surface concentration varied by over four orders of magnitude. The fact that over this domain the slopes of the linear pluts in Figure 3 are linearly related to $k_{\mathrm{H}}^{-1}$ is compelling evidence that $\left\{\mathrm{M}+\mathrm{H}^{+}\right\}$is a linear function of bulk amine concentration. LSIMS spectra appear to reflect the Henry's law concentration of amine.

Previous investigators have concluded that surface excess concentration plays a significant role in secondary ion emission [1, 2, 14-16]. Excluding measured constants, the same qualitative conclusion would be drawn from data presented in Figure $4 \mathrm{~b}$. However, detailed quantitative analysis indicates a poor fit between secondary ion intensity and surface concentration, at least for the system of volatile $n$-alkylamines and glycerol.

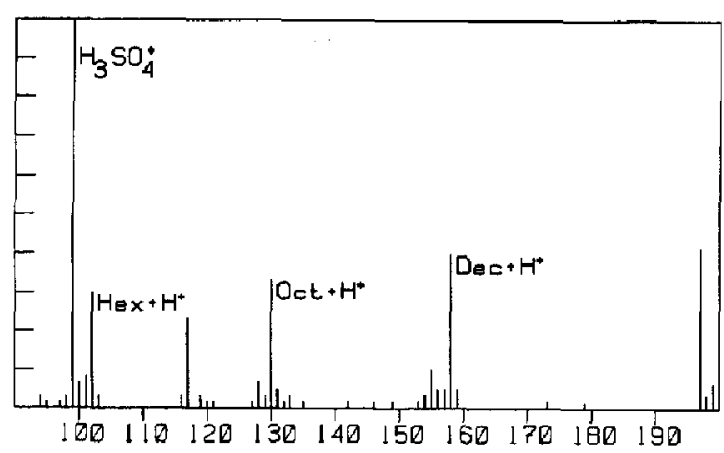

Figure 6. LSIMS spectrum obtained from introduction of the same hexylamine, octylanime, derylamine mixture used for Figure 1 , but introduced onto a sulfuric acid matrix.

The constancy of $d\left\{\mathbf{M}+\mathrm{H}^{+}\right\} / d X$ was tested for the amine analytes by changing to a sulfuric acid matrix. For strongly acidic matrices, gaseous amine molecules are spontaneously protonated as they strike the acid surface, because the reaction of a simple weak base with a strong acid is known to be spontaneous. The concentration of protonated amine on and near the surface then depends only on the balance among rates of adsorption, diffusion, and sputtering, which should be nearly identical for the amines studied. Thus, the relative sensitivity for each amine should be identical according to the conclusions based upon the amine-glycerol data. An equimolar mixture of hexylamine, octylamine, and decylamine was admitted onto a surface of sulfuric acid under the same conditions as those employed when glycerol was used as a matrix. The partial mass spectrum is shown in Figure 6. Within experimental error, the intensities of the protonated amine peaks are identical. This observation substantiates three important points. First, for this simple amine-sulfuric acid system, when analyte concentrations are identical, secondary ion intensities are identical. Second, the assumption that amine chemistry, that is, the chemistry of protonation, is independent of the alkyl chain length is validated. Third, surface activity of the protonated amines is not the dominating factor in determining the relative intensity of emitted secondary ions from sulfuric acid.

The intensity of secondary ions may be insensitive to surface activity because the preponderance of primary ion energy is deposited beneath the surface monolayer, altering the sample composition by damage and subcutaneous chemical reaction. Well-established Monte Carlo methods have been developed to determine the trajectories and energy deposition of high-energy particles impacting on condensed matter [27]. These methods have been widely verified experimentally, mainly as a result of the importance of ion implantation in the semiconductor industry. Following these methods, kinetic energy lost to ionizing molecules as a function of depth was calculated for the experimental conditions reported here $\left(5-\mathrm{keV} \mathrm{Ar}^{+}\right.$, 


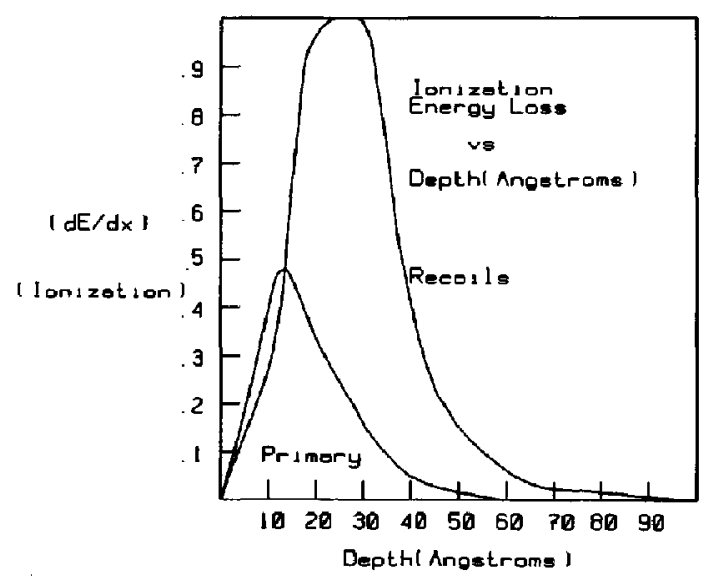

Figure 7. Primary ion kinetic loss versus depth into glycerol sample based on methods described in ref. 27. The distribution is normalized to the maximum value of $d E / d x$ of recoil-induced ionization.

$70^{\circ}$ incidence on glycerol) and is plotted in Figure 7. Roughly $50 \%$ of the primary ion energy is dissipated by sample ionization, the rest being lost by atomic displacement (hole creation) and heating (phonon creation). Figure 7 shows that the primary ion directly creates ions in solution at a rate significantly less than that caused by recoiled atoms in the sample. By both methods of sample ionization, the bulk of energy transfer occurs at levels deeper than the $10-\AA$ surface thickness. Figure 7 does not show where detected secondary ions originate, but simply where ions are formed in solution. It is not unreasonable, however, to believe that at least some secondary ions arise from depths of up to $30 \AA[28,29]$, where the preponderance on ionization occurs and where most of the encrgy is deposited, compared to the surface, where only a small fraction of ionization and energy loss occurs.

\section{Henry's Law Concentration and Secondary Ion Intensity}

It is estimated that 540 glycerol molecules are sputtered in each sputtering event $[9,30]$. The probability $p$ that at least one amine molecule is even present in a random collection of 540 molecules from a solution can be derived from a Poisson distribution, eq 5.

$$
p=1-\exp (-540 X)
$$

where $X$ is the amine mole fraction. For a concentration of $0.1 \mathrm{ppm}$, this probability is $5.4 \times 10^{-5}$. In contrast, the upper axis of Figure 2 shows that at a Henry's law mole fraction of $0.1 \mathrm{ppm}$, the fraction of the total secondary ion current carried by protonated ocytlamine is about $23 \%$; data for the other amines are consistent with this. It is difficult to rationalize the fact that the data indicate that $23 \%$ of the secondary ion

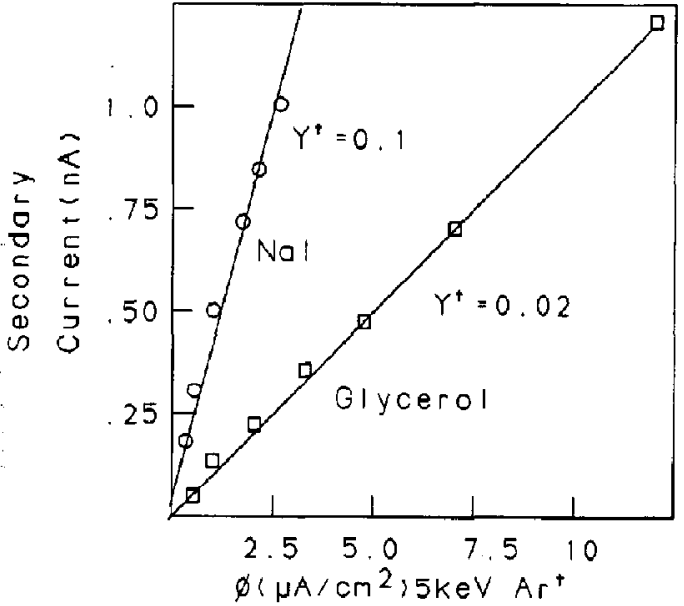

Figure 8. Plot of total secondary ion current accelerated to 8 $\mathrm{keV}$ and focused into the electrostatic analyzer versus $5-\mathrm{keV}$ $\mathrm{Ar}^{+}$primary ion current density. The electrostatic analyzer was wired as a Faraday cup for these measurements.

current is carried by protonated amine when the probability of even finding an amine molecule in any sputtering event is $5.4 \times 10^{-5}$. Either the secondary ion yield of glycerol is much lower than that of amine dissolved in glycerol or the precursors to the secondary protonated amines detected are some species other than free-base amine. The former possibility can be tested directly.

To be consistent with the data, the secondary ion yield of glycerol would have to be less than $1.8 \times 10^{-4}$ and the secondary ion yield from volumes containing an amine molecule unity. Secondary ion yields of many inorganic compounds are well known. In particular, the secondary ion yield of sodium halides subject to $5-\mathrm{keV} \mathrm{Ar}^{+}$is known to be 0.1 [31]. This figure for secondary ion yield can be used as a reference for determination of secondary ion yield from glycerol. All that is necessary is to wire the electrostatic sector of the mass spectrometer as a Faraday cup and, with a picoammeter, measure the total secondary ion current under the same set of primary and secondary source conditions for both reference and sample. Care must be taken that the secondary ion focusing conditions are such that only secondary ions from the target are measured. With $\mathrm{NaI}$ as a reference, this restriction is easily met, because light is emitted from the target where it is struck by the primary ion beam and can be easily observed. Figure 8 shows plots of secondary ion current versus primary 5-keV $\mathrm{Ar}^{+}$for both $\mathrm{NaI}$ and glycerol samples. From the slopes in Figure 8, it is clear that the secondary ion yield from glycerol is $20 \%$ that of $\mathrm{NaI}$ for the conditions employed here, so that the absolute secondary ion yield of pure glycerol is $(0.1)(0.2)=0.02$. This is two orders of magnitude greater than the maximum ion yield of $1.8 \times 10^{-4}$ that can be ascribed to glycerol and still permit free-base amine to be the 
direct precursor to the secondary protonated amine detected. In other words, the secondary ion yield of glycerol is too high to be used as a rationale for the discrepancy between the Henry's law concentration of free-base amine and the fraction of secondary ion current carried by protonated amine. The preponderance of secondary protonated amine detected does not arise directly from free-base amine, but rather from some other precursor whose concentration is not specified by Henry's law.

The most likely candidates to be precursors to secondary protonated amines are amines protonated in solution. That is, evidence indicates that aminecharacteristic secondary ions are "preformed" in solution prior to the sputtering events that made them secondary ions. This does not necessarily imply that a principal requisite for secondary ion emission is existence in solution as an ion. The evidence does indicate that near the surface of a glycerol solution under primary ion bombardment, bases are predominantly protonated.

It is likely that the concentration of protonated amine is determined by kinetic constraints rather than solution-phase ionic equilibria. Ionic equilibrium equations would predict a square root relationship between protonated amine and free-base amine concentrations. The LSIMS data do not follow this prediction. Furthermore, Connolly and Orth [6] showed a lack of correlation between equilibrium ion concentration in bulk solution and secondary ion intensity.

If secondary ion emission is dominated by solution-phase chemical kinetics, then the least restricted description of those kinetics is given by eqs 6 and 7 .

$$
\begin{gathered}
\operatorname{Am}(\mathrm{g}) \stackrel{\text { fast }}{\rightarrow} \mathrm{Am}(\mathbf{l}) \\
\mathrm{Am}(\mathrm{l})+X \stackrel{\text { slow }}{\rightarrow} \mathrm{AmH}^{+}
\end{gathered}
$$

where $A m(B)$ and $A m(1)$ represent gaseous and dissolved amine, respectively, and $\mathrm{X}$ is some protonating agent. Protonation of the amine (eq 7) must be the rate-determining step in the process of converting gas-phase amine molecules to protonated amines in glycerol solution. Were reaction 6 the rate-determining step to protonation, then the protonated amine relative abundances would be independent of Henry's law. This type of behavior is evident in Figure 6, where sulfuric acid was used as a matrix. When glycerol is used as a matrix, the situation is different.

Glycerol, unlike sulfuric acid, cannot spontaneously protonate amines and requires a surrogate protonating agent. The strongest acid that is stable in glycerol is protonated glycerol. It is likely that protonated glycerol is formed, exists, and is consumed in solution as described by eqs 8-10.

$$
\begin{aligned}
& \mathrm{GH}+\phi\left(\mathrm{Ar}^{+}\right) \stackrel{k_{1}}{\rightarrow} \mathrm{G}^{*} \stackrel{\text { fast }}{\rightarrow} \mathrm{GH}_{2}^{+}+\mathrm{G}^{-} \\
& \mathrm{GH}_{2}^{+}+\phi\left(\mathrm{Ar}^{+}\right) \stackrel{k_{2}}{\rightarrow} \text { loss (sputtering) }
\end{aligned}
$$

$$
\mathrm{GH}_{2}^{+}+\mathrm{G}^{-} \stackrel{k_{\mathrm{rc}}}{\rightarrow} \text { loss (recombination) }
$$

where $\mathrm{GH}$ represents neutral glycerol, $\phi\left(\mathrm{Ar}^{+}\right)$represents primary ion current density, and $G^{*}$ represents a praduct formed by primary ion damage to glycerol.

In a single sputtering event, in which large numbers of positive and negative jons are generated, the rate of recombination is very large. In chemical kinetics, this is known as a cage effect; cage-effect kinetics are far too fast to be observed here. For those few ions that escape the cage and go into solution, subsequent recombination is diffusion-limited and slow for two reasons. First, the diffusion coefficients in glycerol are small-about four orders of magnitude smaller than in water. Using well-known methods to estimate diffusion-limited rate constants [32], an upper limit for the recombination rate constant in glycerol, based on diffusion-limited rate constants in water, is found to be about $10^{6} \mathrm{M}^{-1} \mathrm{~s}^{-1}$, a relatively small value. Second, the concentration of such damage-originated ions is likely to be low because of the number that suffer recombination within the sputtering volume. For example, if concentrations of protonated and deprotonated glycerol are each $10^{-4} \mathrm{M}$ outside the cage, the loss rate of each species would be about $1 \% \mathrm{~s}^{-1}$. Compared with a surface erosion rate of 2.2 monolayers/per second at a primary ion current density of 0.5 $\mu \mathrm{A} / \mathrm{cm}^{2}$, the effect of recombination should be negligible.

By asserting that the rate of recombination is negligible compared to the rate of sputtering, the steadystate concentration of protonated glycerol can be stated explicitly as

$$
\left[\mathrm{GH}_{2}^{+}\right]=k_{1} / k_{2}
$$

Amine molecules should be protonated by a reaction similar to that of recombination,

$$
\mathrm{Am}+\mathrm{GH}_{2}^{+} \stackrel{k_{\mathrm{p}}}{\rightarrow} \mathrm{AmH}^{+}+\mathrm{GH}
$$

The rate of formation of protonated amine in solution is given by

$$
\frac{d\left[\mathrm{AmH}^{+}\right]}{d t}=k_{\mathrm{p}}[\mathrm{Am}]\left[\mathrm{GH}_{2}^{+}\right]
$$

Presumably, protonation of amine is diffusion-limited, with a rate constant similar to that for recombination of glycerol ions. The amine concentrations studied here are very low. For example, the concentration of octylamine at an equilibrium partial pressure of $10^{-5}$ torr is $6 \times 10^{-5} \mathrm{M}$. At such low amine concentrations, amine protonation does not significantly affect the steady-state concentration of protonated glycerol. Consequently, combining eqs 1,11 , and 13 , the rate of amine protonation is given by

$$
\frac{d\left[\mathrm{AmH}^{+}\right]}{d t}=\frac{k_{\mathrm{p}} k_{1}\left(k_{\mathrm{H}}^{-1} P_{\mathrm{Am}}\right)}{k_{2}}
$$

where $P_{\mathrm{Am}}$ is the partial pressure of amine. 


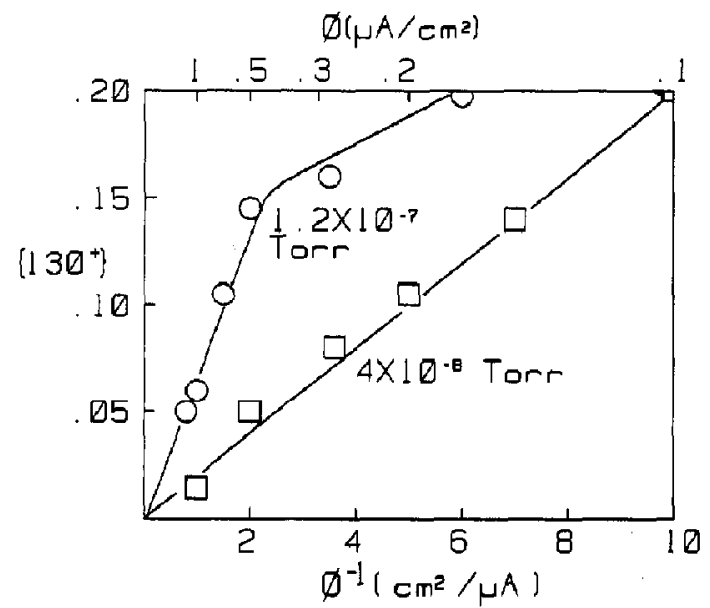

Figure 9. Plot of $\left\{\mathrm{M}+\mathrm{H}^{+}\right\}, m / z$ 130, from octylamine versus inverse primary ion current density. Upper horizontal axis shows corresponding primary ion current density.

If the major source of loss of protonated amine is sputtering and $k_{\mathrm{s}}$ is the sputtering constant, the loss rate of protonated amine is given by the equation

$$
-\frac{d\left[\mathrm{AmH}^{+}\right]}{d t}=k_{\mathrm{s}} \phi\left[\mathrm{AmH}^{+}\right]
$$

Assuming that the rates of amine protonation and loss of protonated amine are equal, that is, a steady. state assumption, the concentration of protonated amine is given by the equation

$$
\left[\mathrm{AmH}^{+}\right]=k_{\mathrm{p}} k_{1}\left(k_{\mathbf{H}}^{-1} P_{\mathrm{Am}}\right) / k_{2} k_{\mathrm{s}} \phi
$$

Equation 16 is consistent with the results presented in Figures 2, 3, and 5, assuming a linear relationship exists between the concentration of protonated amine and the relative abundance of secondary protonated amine. A linear relationship should and does exist between the relative abundance of protonated amine and amine pressure. A surprising feature of eq 16 is the prediction of an inverse relationship between protonated amine relative abundance and primary ion current density. Figure 9 shows that this is the case for protonated octylaminc. At high primary ion current density and low octylamine partial pressure, an inverse relationship indeed exists between protonated amine relative abundance and primary ion current density. The plot in Figure 9 is limited to relatively low pressures because, as can be seen from Figure 2, for constant sensitivity, $\left\{130^{+}\right\}$must not exceed 0.2 .

The inverse relationship between relative abundance and primary ion current density is less satisfactory at low primary ion current density and higher amine pressure. At higher amine concentrations, the rate of amine protonation might reasonably approach the rate of protonated glycerol loss by sputtering, particularly at low primary ion current density. Thus, the steady-state assumption used to derive eq 14 is dubious at high pressure and low current density. In general, the manner in which eq 16 fails at low primary ion current density and high amine pressure is consistent with the limits of the steady-state approximations employed in the derivation of eq 16.

The most compelling argument to support the viability of reaction 12 as a protonating mechanism is that it is analogous to the gas-phase proton-transfer reaction that describes chemical ionization. Reaction 12 is rarely a significant factor in protonation in the liquid phase because the concentration of protonated solvent is so low. Because of this, Sunner and coworkers concluded that the gas-phase counterpart of reaction 12 was the predominant protonation mechanism responsible for generation of secondary protonated solute molecules in FAB/MS experiments [33, 34]. The major differences between the conclusions drawn from this work and those of Sunner et al. are in where and when reaction 12 occurs. The evidence presented here indicates that the predominance of solute protonation occurs in solution. For amine protonation to occur during sputtering. that is, in the gas phase, free-base amine molecules in solution would have to be the direct precursors to the secondary protonated amines detected. This possibility is refuted because relative abundances of secondary protonated amines measured in this work are orders of magnitude larger than can possibly be accounted for by free-base amine concentration in solution. Whether secondary amine ions must be "preformed" in solution is not clear. It is clear, however, that the preponderance of secondary protonated amines detected in this work must have arisen from ions formed in solution.

\section{Conclusions}

In this paper, we present a system that can be used to study, in a quantitative way, relationships between secondary ion intensity and various physical properties of the sample solutions. The range of values for constants defining the various sample systems appears to be larger than any estimates of experimental error and effects of reactions tangential to secondary ionization. With the explicit assumptions listed in the paper, the results of the study indicate that secondary ion emission is governed by the kinetics of reactions near, but below, the surface. Evaluation of the rate constants for the kinetics of eqs $7-16$ is currently under way. Additional reactions such as free radical reactions could be included if indicated in the LSIMS spectra.

The solution kinetics described here are limited to volatile amines introduced onto glycerol under primary ion bombardment. The kinetics describe competition between protonated amine formation and loss for systems in which there is a constant source of amine that fixes the free-base amine concentration, 
regardless of the concentration of protonated amine. Solutions consisting of fixed quantities of nonvolatile solute are subject to the same chemical reactions, but the differential equations relating concentrations of free base and protonated solute are different. For example, there is no straightforward way to distinguish protonated solute concentration from free-base solute concentration when only the total analytical concentration of solute is known.

When free-base amine concentration is determined by amine partial pressure, the data lead to the conclusion that free-base amines cannot be the direct precursors to the majority of secondary protonated amines detected. Extensive protonation appears to accur in solution, probably involving a reaction between a charged matrix species and a free-base solute molecule, before sputtering. In our view, the question of whether preexistence as ions in solution is a prerequisite for secondary ion emission is vacated by the answer that basic species are overwhelmingly protonated if they are in the region from which secondary ions are emitted. Thus, for a solute to give rise to a solute-characteristic positive secondary ion, the protonated or otherwise positively charged form of the solute must be stable in glycerol solution. If the positively charged form of the solute is chemically unstable, the solute is consumed in the sputtering region before it can be emitted as a secondary ion.

Results presented here are consistent in many ways with the results of others. The functional behavior of secondary ion intensity with respect to pressure is similar to the behavior of secondary ion intensity with respect to concentration reported by others. The mechanism of protonation is identical to that proposed by others, except that we believe that it occurs prior to the individual sputtering events that give rise to secondary ions.

Conclusions drawn here must be qualified. Correlations are not exclusive. For example, the qualitative increase in sensitivity with surface activity and $k_{\mathrm{H}}^{-1}$ is coincidental. Furthermore, the data presented here do not guarantee a universal correlation of sensitivity with $k_{\mathrm{H}}^{-1}$, although they do not preclude a predominating effect of surface activity on secondary ion emission, except by coincidence. For solutes dissolved in glycerol, a qualitative correlation exists between diffusion coefficients and alkyl chain lengths, for example. The effects of diffusion are difficult to discern, but they may be responsible for the apparent correlation between secondary ion intensity and surface activity reported by others. The apparent absence of diffusive effects when solutes are introduced via the gas phase may explain the differences between the results reported here and those of others.

\section{Acknowledgments}

I am indebted to W. H. Christie, C. P. Leibman, R. F. Porter, K. D. Cook, F. W. Rollgen, and P. Williams for helpful discussions. Insights gained from the initial reviewers of this paper are gratefully acknowledged. This work was funded by the U.S. Department of Energy, Office of Basic Energy Sciences, under contract DE-AC05-84OR21400 with Martin Marietta Energy Systems, Inc.

\section{References}

1. Barber, M.; Bordoli, R. S.; Elliot, R. D.; Sedgwick, R. D.; Tyler, A. N. Anal. Chem. 1982, 54, 645A-657A.

2. Lacey, M. P.; Keough, T. Rapid Commun. Mass Spectrom. 1989, 3, 46-50.

3. Field, F. H. I. Phys. Chem. 1982, 86, 5115-5123.

4. Cole, R. B.; Guenat, C.; Hass, J. R.; Linton, R. W. Anal. Chem. 1987, 59, 1930-1937.

5. Gale, P. J.; Bentz, B. L.; Chait, B. T.; Field, F. H.; Cotter, R. J. Anal. Chem. 1986, 58, 1070-1076.

6. Connolly, M. J.; Orth, R. G. Anal. Chem. 1987, 59, 903-908.

7. Groenewold, G. J.; Todd, P. J. Anal. Chem. 1985, 57, 886-890.

8. Berry, R. S.; Rice, S. A.; Ross, J. Physical Chemistry; Wiley: New York, 1980; pp 914-916.

9. Todd, P. J.; Groenewold, G. J. Anal. Chem. 1986, 58, 895-899.

10. Crank, J. D. The Mathematics of Diffusion; Clarendon Press: Oxford, 1979; p 137.

11. Evanoff, J.; Harris, W. E. Can. J. Chem. 1978, 56, 574-577.

12. Hiemenz, P. C. Principles of Colloid and Surface Chemistry; Marcel Dekker: New York, 1986; pp 386-391.

13. Adamson, A. W. Physical Chemistry of Surfaces; Interscience: New York, 1967; pp 568-572.

14. Barber, M.; Bordoli, R. S.; Elliot, G. J.; Sedgwick, R. D.; Tyler, A. N. J. Chem. Soc., Faraday Trans. 1 1983, 79, 1249-1255.

15. Ligon, W. V.; Dorn, S. B. Int. J. Mass Spectrom. Ion Processes 1984, 57, 75-90.

16. Allmaier, G. M. Rapid Commun. Mass Spectrom. 1988, 2, 74-76.

17. McBain, J. W.; Swain, R. C. Proc. Roy. Soc. London 1936, A154, 608-618.

18. Weast, R. C., Ed. Handbook of Chemistry and Physics, 46th ed., Chemical Rubber Co.: Cleveland, 1965; p F-43.

19. Wong, S. S.; Rollgen, F. W. Nucl. Instrum. Methods 1986, B14, 436-447.

20. Walder, R.; Franklin, J. L. Int. J. Mass Spectrom. Ion Phys. 1980, 36, 85-112.

21. Ralson, A. W.; Selby, W, M.; Pool, W. O.; Potts, R. H. Ind. Eng. Chem. 1940, 32, 1093-1094.

22. Todd, P. J.; Glish, G. L. Int. J. Mass Spectrom. Ion Processes 1984, 61, 215-230.

23. Todd, P. J. Org. Mass Spectrom. 1988, 23, 419-424.

24. Christie, A. O.; Crisp, D. J. I. Appl. Chem. 1967, 17, 11-14.

25. Meot-Ner (Mautner), M. In Gas Phase Ion Chemistry; Bowers, M. T., Ed.; Academic: New York, 1979; Vol. 1, PP 197-271.

26. Leibman, C. P.; Todd, P. J.; Mamantov, G. Org. Mass Spectrom. 1988, 23, 634-642.

27. Ziegler, J. F.; Biersack, J. P.; Littmark, U. The Stopping and Range of lons in Solids; Pergamon: New York, 1985.

28. Todd, P. J. In SIMS V. Proceedings of the Fifth Inlernational Conference on Secondary Ion Mass Spectrometry; Benninghoven, A., Ed., Springer-Verlag: New York, 1986; pp 54-56.

29. Sunner, J.; Ikonomou, M, G.; Kebarle, P. Int. J. Mass Spectrom. Ion Processes 1988, 82, 221-237. 
30. Jiang, L. F.; Barofsky, E.; Barofsky, D. F. Proceedings of the 36th Annual Conference on Mass Spectrometry and Allied Topics, June 5-10, 1988; pp 1209-1210.

31. Carter, G,; Colligan, J. S. Ion Bombardment of Solids; American Elsevier: New York, 1968; pp 64-65.

32. Amdur, I.; Hammes, G. G. Chemical Kinetics: Principles ant
Related Topics; McGraw-Hill: New York, 1966; p 148.

33. Sunner, J. A.; Kulatunga, R,; Kebarle, P. Anal. Chem. 1986, 58, 1312-1316.

34. Sunner, J. A.; Kulatanga, R.; Kebarle, P. Anal. Chem. 1986, $58,2009-2014$. 